DOI:

Article Type: Full Paper

\title{
Quantifying a Heterogeneous Ru Catalyst on Carbon Black using ADF STEM.
}

A. M. Varambhia, L. Jones, A. De Backer, V. T. Fauske, S. Van Aert, D. Ozkaya and P. D. Nellist*

A. M. Varambhia, Dr. L. Jones, Prof. P. D. Nellist

Department of Materials, 13 Parks Road, Oxford, OX13PH, UK

Email: Peter.Nellist@materials.ox.ac.uk

Dr. A De Backer, Prof S. Van Aert

EMAT, University of Antwerp, Groenenborgerlaan 171, B-2020 Antwerp, Belgium

Dr V. T. Fauske

Department of Physics, NTNU, 4791 Trondheim, Norway

Dr. D. Ozkaya

Johnson Matthey Technology Centre, Sonning Common, Reading, RG4 9NH, UK.

Abstract: $\mathrm{Ru}$ catalysts are part of a set of late transition metal nanocatalysts that have garnered much interest for catalytic applications such as ammonia synthesis and fuel cell production. Their performance varies greatly depending on their morphology and size, these catalysts are widely studied using electron microscopy. Using recent developments in Annular Dark Field (ADF) Scanning Transmission Electron Microscopy (STEM) quantification techniques, a rapid atom counting procedure was utilized to document the evolution of a heterogeneous Ru catalyst supported on carbon black. Areas of the catalyst were imaged for approximately 15 minutes using ADF STEM. When the Ru clusters were exposed to the electron beam, the clusters changed phase from amorphous to crystalline. To quantify the thickness of the crystalline clusters, two techniques were applied (simulation and statistical decomposition) and compared. These techniques show that stable face centred 
cubic crystal structures in the form of rafts, between 2 and 8 atoms thick, were formed after the initial wetting of the carbon support.

Keywords: Ru catalysts, heterogeneous catalyst rafts, STEM, image quantification

\section{Introduction}

Ru-based catalysts are used in many applications such as ammonia production, catalytic converters and fuel cells ${ }^{[1]}$. Theoretical modelling shows that ammonia synthesis over $\mathrm{Ru}$ is an even more surface sensitive reaction compared to alternative catalysts such as iron ${ }^{[2][3]}$. Over the years, there has been increasing emphasis on commercial Ru/C catalysts ${ }^{[3]-[6]}$. Such catalysts show promising potential over the current common industrial catalysts ${ }^{[1][6][7]}$. Similarly, discoveries of the potential of $\mathrm{Ru}$ catalysts have been extended to other areas (such as CO oxidation ${ }^{[6][8]}$ ). The activity of catalyst nanoparticles depends on the morphology of their exposed surface, which itself can strongly depend on the support material ${ }^{[2]}$. However determining their morphology is a severe characterisation challenge.

There are several papers that have presented theoretical and experimental studies of $\mathrm{Ru}$ nanoparticles $^{[1][9]-[11]}$. Two-dimensional raft-like structures in supported heterogeneous Ru catalysis were first reported using conventional TEM image contrast by Prestridge et al. ${ }^{[12]}$. In their paper it was concluded that due to the uniformly low contrast from the Ru crystals, the crystals observed were rafts. It was later pointed out that this method of determining particle thickness can be misleading ${ }^{[13][14]}$ because contrast is dependent on the orientation of the metal crystal with respect to the electron beam. Additionally, a microdiffraction study of Au-Ru nanoparticle patterns showed that some patterns could not be attributed to any known structure $^{[15]}$. For these patterns, agreement could be found if a raft-like morphology was assumed. 
With the greater availability of aberration corrected electron microscopes it is now routinely possible to achieve atomic resolution for many materials. Annular dark-field (ADF) imaging in the scanning transmission electron microscope (STEM) provides images with contrast that depends strongly on atomic number (Z-contrast) and also depends on the sample thickness in a monotonic way ${ }^{[16]}$. These properties make it highly suitable for the study of supported nanoparticle catalysts with single atom detection possible ${ }^{[17]}$.

Recently, there have been developments in methods to accurately calibrate the ADF detector to make quantitative measurements of electron scattering in the imaging process ${ }^{[18]}$. Measurement of the strength of the electron scattering allows the sample thickness to be determined, and it is now possible to obtain three-dimensional structural models from image intensities $^{[19]}$. An alternative approach is to use the distribution of measured scattered intensities in a statistical analysis, making use of the discrete nature of atoms, to determine sample thickness ${ }^{[20]}$. In this paper both a calibrated intensity quantification approach ${ }^{[21]}$ and a statistical analysis approach ${ }^{[22]}$ are used to provide evidence for rafts in the $\mathrm{Ru} / \mathrm{C}$ system. The rafts are initially seen to be disordered, but start to crystallise under electron irradiation. The combinational approach unlocks the potential to study metal-support interactions for such systems. Combining this with the theoretical modelling can lead to a detailed insight into the fundamental kinetics of catalysts with their support material.

\section{Sample preparation, data acquisition and quantification methods}

\subsection{Ru Catalyst Synthesis}

The carbon supported Ru catalyst was supplied by Johnson Matthey Technology Centre and

prepared using an adaptation of an established deposition precipitation method ${ }^{[37]}$ starting with $\mathrm{RuCl}_{3}$. The final product was achieved by reducing in $5 \% \mathrm{H}_{2} / \mathrm{N}_{2}$ at $100{ }^{\circ} \mathrm{C}$ for $1 \mathrm{~h}$.

\subsection{Imaging Parameters}


The catalyst was imaged using ADF STEM on a JEOL ARM 200F with detector collection angles spanning $69 \mathrm{mrad}$ to $138 \mathrm{mrad}$. Three areas of the Ru catalyst were imaged. These areas, named as R1, R2 and R3, are shown in Figure 1. Initially regions R1 and R2 were observed as being amorphous, whereas R3 had crystalline and amorphous parts. Movies were recorded to observe any possible changes; 33 frames were recorded for R1 and R2, and 44 frames were recorded for R3.

Both areas were imaged for approximately 15 minutes each with a beam current of approximately 35 pA. After applying various corrections, the three areas in Figure 1 were then quantified for two key features: crystal lattice system and thickness.

\subsection{Correcting Experimental Parameters for Quantification}

In order to quantify the images accurately, some experimental calibrations were made before acquiring the data. The general procedure applied to these images is summarised as follows. First an image of the detector sensitivity is acquired ${ }^{[23]}$, this is done to measure variations in the detector sensitivity. Most simulation software assume a perfectly uniform detector, whereas in an experimental situation, detector sensitivity varies greatly ${ }^{[23]}$. To correct for this, an approach proposed by Martinez et $\mathrm{al}^{[24]}$ was used to normalise the images using an electron flux weighting. With effective uniform detector linearity, the measured intensities can then be directly correlated to atom counts using a previously published method ${ }^{\text {[25]-[27] }}$.

After the detector sensitivity was normalised, the images were recorded and further corrections were applied. One of these corrections is normalisation of the electron current at the detector. The current of cold field emission guns decay throughout the imaging process. Due to this, a decrease in the image intensity is seen, thus giving an incorrect estimation of the atomic columns during quantification. The current was recorded at different intervals and then factored into the quantification to compensate for this decay. 
Finally the images were scan-drift corrected using a technique developed by Jones et al. ${ }^{[28]}$. Scanning distortions can lead to errors such as imprecisely mapping peak positions and incorrectly correlating peak positions between sets of images for comparisons. They can also lead to incorrect intensity quantification. The correction ensured that the relative areas within the analysis regions remained consistent between frames.

\section{Results and Discussion}

Structure type (nanoparticle, cluster, rafts, porous etc), crystal lattice system (fcc, bcc, amorphous etc), particle size and support are some of the parameters that affect the performance of a catalyst. For Ru nanocatalysts the performance appears to strongly depend on the crystallography and the exposed surface plane, even more so than conventional catalysts $^{[1]-[3]}$. Additionally, the type of support material also plays a key role in determining particle crystallography ${ }^{[29]}$ and also morphology ${ }^{[2]}$.

\subsection{Determining the Crystal Lattice System}

Determining the crystal lattice system of a catalyst is important as it affects catalyst performance. However, it is also a parameter that is required for the thickness quantification process. To explore this further, a set of frames from the recorded sequence were chosen and analysed. Three consecutive frames for each region, with a maximum drift rate of 1 pixel between the three frames, were chosen and averaged. The frames that were chosen originate from the latter stages of the movie, where drift was fairly stable ensuring that the column positions were relatively drift free and the atomic column positions could be labelled reliably for structural analysis.

A map of vectors linking the atomic column locations with their respective bond angles was created using an in-house software (Figure 2). In the figure, the measured angles between the outgoing yellow and blue vectors are labelled. From examination of the distribution of angles 
in Figure 2d, it can be seen that for R1 the distribution peaks close to $90^{\circ}$, with a mean of $88.13^{\circ}$ and a standard error of $1.12^{\circ}$, whereas for R2 and R3 the angles peak at just under $110^{\circ}$, with means of $106.11^{\circ} \pm 1.21^{\circ}$ and $103.52^{\circ} \pm 0.98^{\circ}$ respectively where the \pm quotes the standard error. Such angles are characteristic of projections of a face-centred cubic (fcc) structure, with the $<100>$ projection giving $90^{\circ}$ and the $<110>$ projection giving $109^{\circ}$.

Modulus of the blue and yellow vectors were also measured (Figure 2e). In an fcc structure the vector modulus of R2 and R3 (assuming $<110>$ orientations) should be related to R1 $(<100>$ orientation) by a factor of $\sqrt{6} / 2$. The mean vector moduli and standard deviations were $2.08 \AA \pm 0.02 \AA, 2.26 \AA \pm 0.03 \AA$, and $2.31 \AA \pm 0.02 \AA$ for regions $\mathrm{R} 1, \mathrm{R} 2$, and $\mathrm{R} 3$ respectively. The expected inter-peak spacing using the fcc Ru lattice parameters from refs ${ }^{\text {[7] [11] }}$ for both orientations are also shown in Figure 2e.

The angle and peak distance measurements are suggestive of an fcc structure, but we note that the distance measurements in particular show significant deviation from the expected values. Deviations from the expected angle and vector moduli may be explained by a number of factors such as the inclination of the carbon support surface to the incoming beam direction, the nearest neighbour chosen by the peak assignment algorithm ${ }^{[19]}$ and defects in the $\mathrm{Ru}$ structure. The structures are clearly still somewhat from equilibrium, and may be influenced significantly by the C support.

The proposal of fcc $\mathrm{Ru}$ is also supported by theoretical calculations and experiments performed by a number of groups. One of the first reports of fcc Ru synthesis was by Kusada et al ${ }^{[6]}$. Additionally; DFT calculations, experiments and fcc Ru nanoparticle synthesis by Zhao et $\mathrm{al}^{[11]}$ show that under certain pressure-temperature conditions it is possible to form fcc Ru. Furthermore, recent synthesis of meta-stable Ru nanoparticles without a support 
material by Abo-Hamed et al ${ }^{[7]}$ also indicates that crystalline phase observed in Figure 1 may be fcc.

\subsection{Quantifying the Thickness within the Regions of Interest}

Once the observed regions have been determined as fcc, the image intensities can be used to measure the thickness of the nanostructures. The thickness of the catalyst within the regions of interest (Figure 1) was quantified using two methods: comparison to simulations and statistical decomposition. In the comparison to simulations method, the total intensity of atomic columns within the images were integrated using Voronoi cells ${ }^{[30]}$. The integrated intensity can be shown to be a scattering cross-section for the column, and this quantity is robust to many imaging parameters ${ }^{[21]}$. The measured cross-sections were then compared to a reference simulation library of a Ru crystal using the method described in Refs ${ }^{[19][21]}$. A Ru crystal was simulated using the multislice method with the $\mu$ STEM software ${ }^{[31]}$ at increasing column thicknesses, for up to 10 atom column thickness, using 30 phonons and a 2048x2048pix supercell tiled 8 times in $\mathrm{x}$ and y. Figure 3 shows that the simulated crosssection plotted as a function of number of atoms in a column is relatively insensitive up to 5 atoms to whether $\mathrm{a}<100>$ or $<110>$ viewing direction is used for the fcc lattice.

The scattering cross-sections from the simulation were then compared to the measured crosssections of the atomic columns within the regions of interest using Absolute integrator software $^{[19]}$. Previous work has demonstrated that this type of quantification method can have a typical error of \pm 1 atom sensitivity for columns up to 20 atom thicknesses ${ }^{[26][32]}$.

In the statistical decomposition approach, the distribution of scattering cross-sections is decomposed into a small number of overlapping Gaussian distributions, where the optimal number of Gaussian components is selected using an Integrated Classification Likelihood (ICL) approach ${ }^{[32][33]}$. This statistical decomposition is used to assign atom counts to the atom 
columns .This technique relies only on the relative intensities from the experimental images and thus does not depend on accurate calibration of microscope parameters. It does, however, require a sufficiently large number of measurements combined with a sufficiently high precision in measurements that could be limited by noise, microscope instabilities etc ${ }^{[22]}$. Here, both approaches have been used to provide a consistent picture of sample thickness.

For the ICL analysis, cross-sections from a combined set of 3 frames (1 from each region) were used. The fitted Gaussians (and the optimal order selection criterion) from the Gaussian Mixture Model are shown in Figure 4. The arrow indicates that the optimal number of components was 7.

Having assigned a number of atoms to a particular Gaussian component using the ICL approach, the mean cross-section for that Gaussian can be compared with the cross-section derived from an image simulation approach, as shown in Figure $\mathbf{5}$ which shows a discrepancy between the two methods. The scattering cross-sections from ICL increase linearly with thickness whereas the room temperature $\mathrm{Ru}$ image simulation does not. The non-linearity in the simulated cross-sections seen in Figure 5 arises because of the channelling of electrons along atomic columns ${ }^{[16]}$. As the Ru atoms were constantly moving due to recrystallization (see images movies in the supplementary information), it is likely that they are significantly displaced from perfect alignment, decreasing the channelling. Such displacements can be included in scattering cross-section simulations. By increasing the RMS displacement within simulation to model the disorder of the sample, a more agreeable and realistic match between the two techniques was obtained (also shown in Figure 5). This approach demonstrates how using both simulation matching and statistical analysis can provide a consistent and convincing analysis of sample thickness. 
Using a linear scaling of cross-section with respect to thickness as a good compromise between the library matching and statistical techniques, the cross-sections from the observed areas were converted into three dimensional surface plots, Figure 6. The linear scaling model is by definition insensitive to whether a $<100>$ or $<110>$ orientation is observed since the cross-section is simply proportional to the number of atoms. Some gaps within the plots e.g. $\mathrm{R} 1, \mathrm{t}=121 \mathrm{~s}$, indicate that not all columns could be quantified using peak labelling as the regions at the beginning were still amorphous. The latter plots indicate a raft-like topology for the all regions. This observation confirms a similar hypothesis made by Prestridge et $\mathrm{al}^{[12]}$, but here a robust image quantification approach is used, rather than making qualitative use of convention TEM contrast which was later criticized by Treacy and Howie ${ }^{[14]}$ as likely to be misleading.

Additionally the thickness of the observed regions stabilised after a certain imaging time (Figure 7). For frames (mostly the initial frames) where the thickness could not be resolved due to amorphous regions, the mean thickness was quantified using mean fractional beam current.

Figure 7 shows that whilst imaging, the nanoparticle changes from an amorphous to a crystalline state forming a structure that does initially increase in thickness before the thickness stabilises, but is still raft-like in nature having a thickness that is much smaller than the lateral extent. The stabilisation of the structure may be due to the carbon support as Ru is known to interact strongly with the support material ${ }^{[1]-[3]}$. The support can play a role in not only particle stability but also morphology ${ }^{[2]}{ }^{[13]}$. DFT calculations by Zhao et al. ${ }^{[11]}$ for a slab of Ru crystal that is 6 atoms thick show that an fcc structure can be obtained if some of the carbon atoms from the support occupy the interstitial sites within the Ru structure ${ }^{[11]}$. 
There is also a possibility that a Ru carbide structure may have formed, this structure may be extremely stable and durable under the beam as bulk RuC has been reported to be a highly durable material ${ }^{[34]}$. However the possibility of this occurring is unlikely, as it is believed that Ru has difficulties forming carbides ${ }^{[11][34]-[36]}$.

In summary, the structure observed here is raft-like with fcc crystallography. The results here present a possibility that $\mathrm{Ru}$ rafts can be formed and could have potential implications towards catalytic performance within certain chemical processes. Additionally fcc Ru nanoparticles are reported to have improved catalytic performance over their hcp counterparts $^{[6][7][11]}$.

\section{Conclusions}

Utilising the latest quantification techniques in ADF Microscopy, a Ru catalyst on carbon black support was analysed. Three regions, were recorded for approximately 15 minutes. These regions showed that the Ru particle started off as an amorphous agglomeration (or both crystal/amorphous agglomeration) of atoms and then fully crystallised. After applying scandrift correction and current normalisation the images were quantified. From the vector mapping and angle measurements the crystal structure was suggested to be fcc with a lattice parameter close to those observed previously for fcc Ru. Using two established methods of quantifying thickness it was seen that assuming the absence of electron channelling was important to get agreement between the approaches, which is justified by the large amount of disorder in the particle. It was seen that the regions observed form stable rafts of up to 6 atoms in thickness. This could have interesting implications for catalytic performance as fcc $\mathrm{Ru}$ nanoparticles have been synthesised previously but $\mathrm{Ru}$ rafts have not previously been conclusively demonstrated.

\section{Acknowledgements}


The authors would like to thank the EPSRC and Johnson Matthey for funding this work as part of a CASE-Award studentship. The research leading to these results has received funding from the European Union Seventh Framework Programme under Grant Agreement 312483 ESTEEM2 (Integrated Infrastructure Initiative-I3). We would like to thank Brian Theobald and Jonathan Sharman from JMTC for provision of the samples The authors gratefully acknowledge the Research Foundation Flanders (FWO, Belgium) for funding and for a postdoctoral grant to ADB. The microscope used was funded by the INFRASTRUKTUR Grant 197405 (NORTEM) program of the Research Council of Norway.

\section{References}

[1] N. Saadatjou, A. Jafari, S. Sahebdelfar, Chem. Eng. Commun. 2014, 202, 420.

[2] C.J.H. Jacobsen, S. Dahl, P.L. Hansen, E. Törnqvist, L. Jensen, H. Topsøe, D. V. Prip, P.B. Møenshaug, I. Chorkendorff, J. Mol. Catal. A Chem. 2000, 163, 19.

[3] W. Raróg-Pilecka, E. Miśkiewicz, D. Szmigiel, Z. Kowalczyk, J. Catal. 2005, 231, 11.

[4] G.S. Benner, J.R. Le Blanc, J.M. Lee, H.P. Leftin, P.J. Shires, C.P. Van Dijk, Supplemental Ammonia Synthesis, 1986, US Patent 4,568,532.

[5] BP, Process for the Synthesis of Ammonia Using Catalysts Supported on Graphite Containing Carbon, 1979, US Patent 4,163,775.

[6] K. Kusada, H. Kobayashi, T. Yamamoto, S. Matsumura, N. Sumi, K. Sato, K. Nagaoka, Y. Kubota, H. Kitagawa, J. Am. Chem. Soc. 2013, 135, 5493.

[7] E.K. Abo-Hamed, T. Pennycook, Y. Vaynzof, C. Toprakcioglu, A. Koutsioubas, O.A. Scherman, Small 2014, 10, 3145.

[8] S. Alayoglu, A.U. Nilekar, M. Mavrikakis, B. Eichhorn, Nat. Mater. 2008, 7, 333. 
[9] P. Liu, a. Logadottir, J.K. Nørskov, Electrochim. Acta 2003, 48, 3731.

[10] F.R. García-García, a. Guerrero-Ruiz, I. Rodríguez-Ramos, Top. Catal. 2009, 52, 758.

[11] Z. Zhao, C. Meng, P. Li, W. Zhu, Q. Wang, Y. Ma, G. Shen, L. Bai, H. He, D. He, D. Yu, J. He, B. Xu, Y. Tian, Nanoscale 2014, 6, 10370.

[12] E.B. Prestridge, G.H. Via, J.H. Sinfelt, J:Catal. 1977, 50, 115.

[13] A.K. Datye, A.D. Logan, N.J. Long, J. Catal. 1988, 109, 76.

[14] M.M.J. Treacy, A. Howie, J. Catal. 1980, 63, 265.

[15] J.M. Cowley, R.. Plano, J. Catal. 1987, 108, 199.

[16] P.D. Nellist, S.J. Pennycook, Adv. Imaging Electron Phys. 2000, 148.

[17] P.D. Nellist, S.J. Pennycook, Science. 1996, 274, 413.

[18] J.M. Lebeau, S.D. Findlay, L.J. Allen, S. Stemmer, Phys. Rev. Lett. 2008, 100, 1.

[19] L. Jones, K.E. MacArthur, V.T. Fauske, A.T.J. van Helvoort, P.D. Nellist, Nano Lett. 2014, 14, 6336.

[20] S. Van Aert, A. De Backer, G. Martinez, B. Goris, S. Bals, G. Van Tendeloo, A. Rosenauer, Phys. Rev. B 2013, 87, 064107.

[21] H. E, K.E. MacArthur, T.J. Pennycook, E. Okunishi, A. J. D’Alfonso, N.R. Lugg, L.J. Allen, P.D. Nellist, Ultramicroscopy 2013, 133, 109.

[22] A. De Backer, G.T. Martinez, A. Rosenauer, S. Van Aert, Ultramicroscopy 2013, 134, 23.

[23] K.E. MacArthur, L. Jones, P.D. Nellist, J. Phys. Conf. Ser. 2014, 522, 012018. 
[24] G.T. Martinez, L. Jones, A. De Backer, A. Béché, J. Verbeeck, S. Van Aert, P.D. Nellist, Ultramicroscopy 2015, 159, 46.

[25] S.D. Findlay, J.M. LeBeau, Ultramicroscopy 2013, 124, 52.

[26] J.M. Lebeau, S.D. Findlay, L.J. Allen, S. Stemmer, Nano Lett. 2010, 10, 4405.

[27] J.M. LeBeau, S. Stemmer, Ultramicroscopy 2008, 108, 1653.

[28] L. Jones, H. Yang, T.J. Pennycook, M.S.J. Marshall, S. Van Aert, N.D. Browning, M.R. Castell, P.D. Nellist, Adv. Struct. Chem. Imaging 2015, 1, 8.

[29] W.B. Monosmith, J.M. Cowley, Ultramicroscopy 1983, 12, 177.

[30] A. Rosenauer, T. Mehrtens, K. Müller, K. Gries, M. Schowalter, P. Venkata Satyam, S. Bley, C. Tessarek, D. Hommel, K. Sebald, M. Seyfried, J. Gutowski, A. Avramescu, K. Engl, S. Lutgen, Ultramicroscopy 2011, 111, 1316.

[31] L.J. Allen, A. J. D'Alfonso, S.D. Findlay, Ultramicroscopy 2014, 1.

[32] S. Van Aert, A. De Backer, G.T. Martinez, B. Goris, S. Bals, G. Van Tendeloo, a. Rosenauer, Phys. Rev. B - Condens. Matter Mater. Phys. 2013, 87, 064107.

[33] S. Van Aert, K.J. Batenburg, M.D. Rossell, R. Erni, G. Van Tendeloo, Nature 2011, 470, 374.

[34] N.R. Sanjay Kumar, N. V Chandra Shekar, S. Chandra, J. Basu, R. Divakar, P.C. Sahu, J. Phys. Condens. Matter 2012, 24, 362202.

[35] N.B. Shitova, P.G. Tsyrulnikov, D.A. Shlyapin, P.S. Barbashova, D.I. Kochubei, V.I. Zaikovskii, J. Struct. Chem. 2009, 50, 268.

[36] V.O. Khavrus, E.M.M. Ibrahim, A. Bachmatiuk, M.H. Rümmeli, a. U.B. Wolter, S. Hampel, A. Leonhardt, J. Nanoparticle Res. 2012, 14. 
[37] S.C. Ball, G. Hards, R.L. O'malley, B. R. C. Theobald, D. Thompsett, 2012, Patent number WO 2012017226 
a) R1 and R2 regions

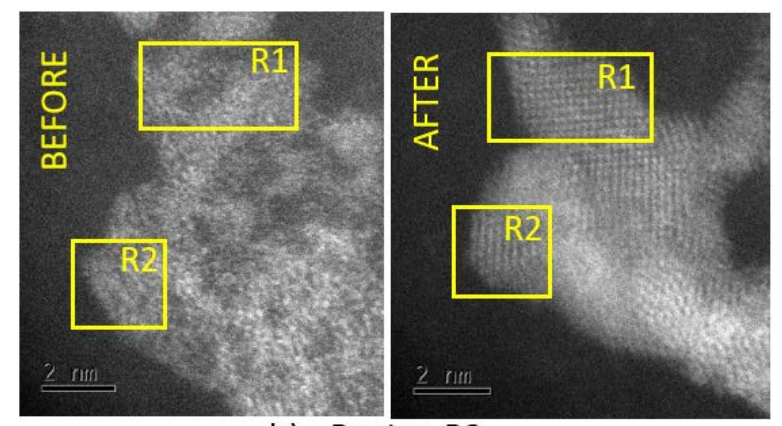

b) Region R3

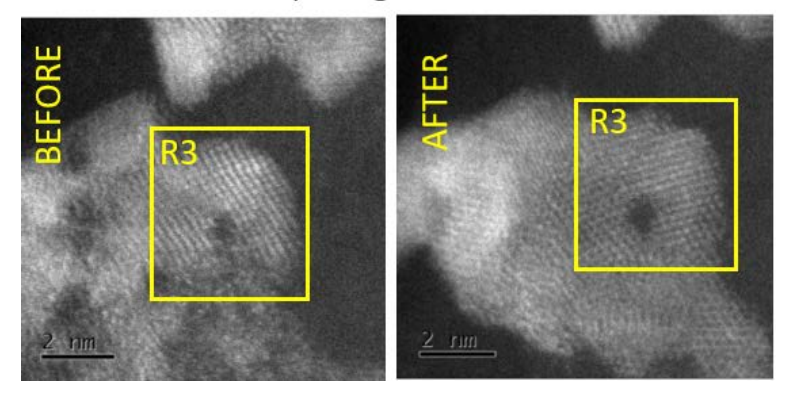

Figure 1. Wide field of view images of the Ru catalyst on carbon black, before and after $\sim 15$ minutes total imaging time, using a 35pA beam and $38 \mu$ s pixel dwell time. The analysis regions are highlighted in yellow. The R1 and R2 were initially amorphous and R3 was partially amorphous. The catalysts had been morphing before the acquisition process started, and hence do not represent the catalyst's initial state. 

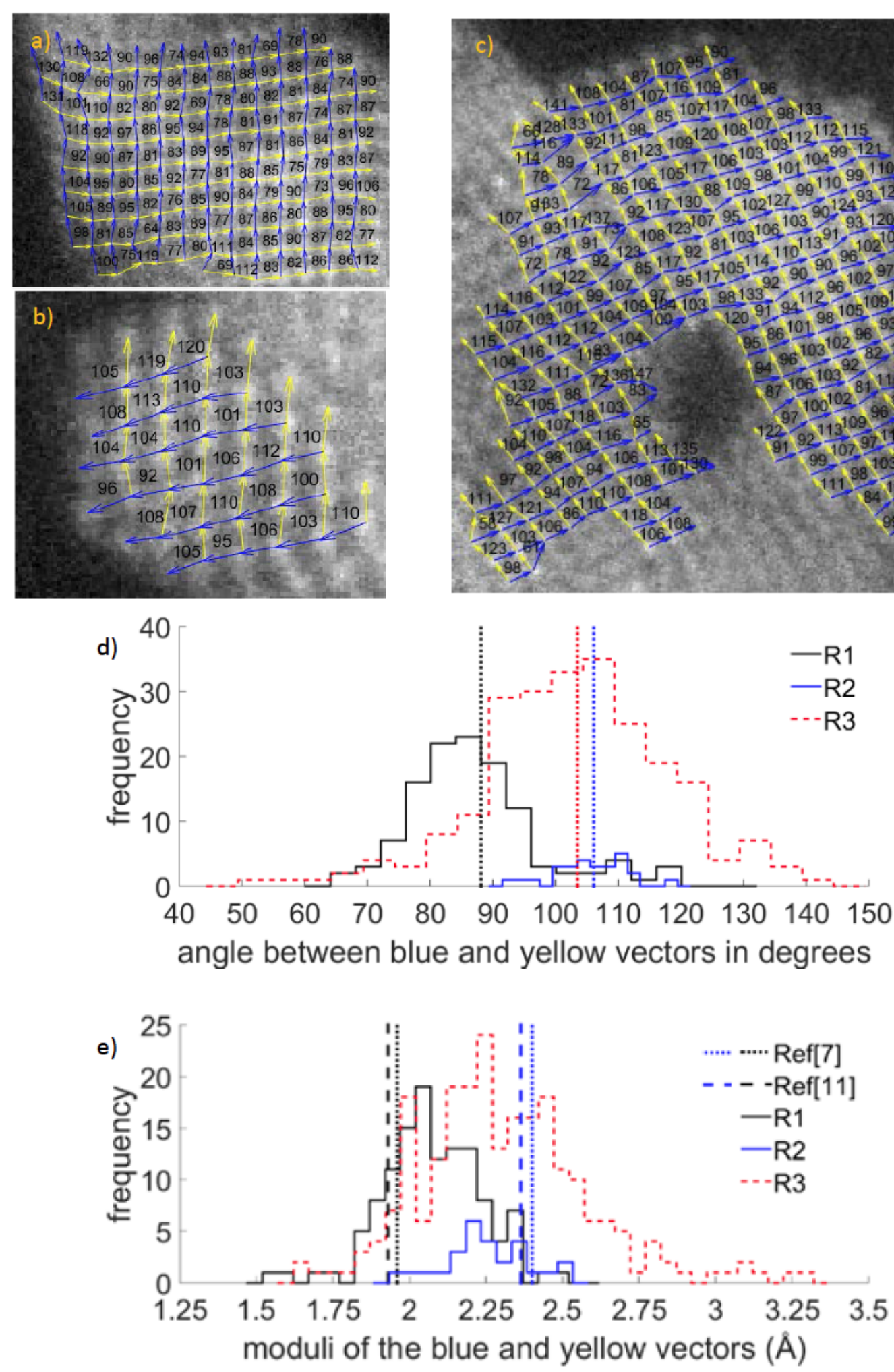

Figure 2. a), b) and c) Enlargements of the areas shown in Figure 1 with atomic-column nearest-neighbour pairings annotated. d) Histogram showing the measured angles and indicated means (vertical dashed lines). e) Histogram of vector lengths with indicated literature lengths for fcc Ru. 


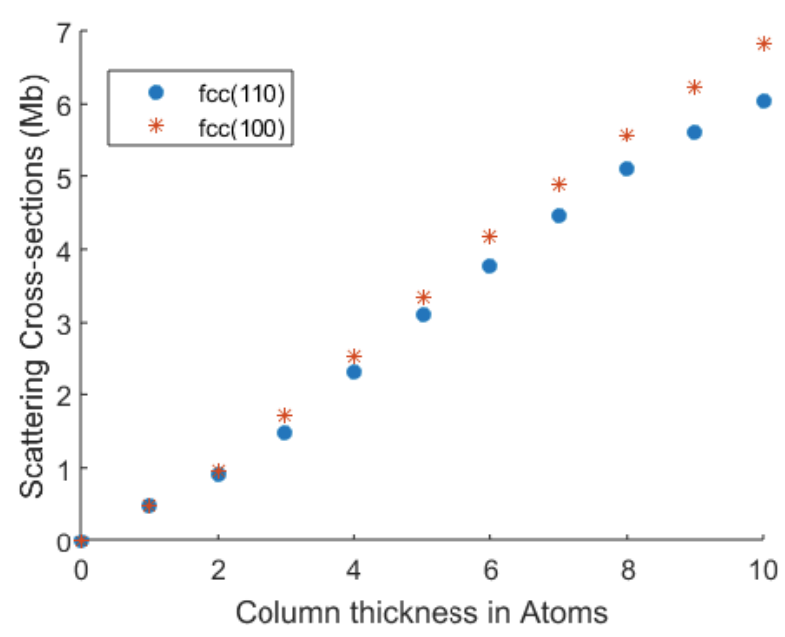

Figure 3. Simulated cross-sections for different Ru crystal orientations. The comparison shows that different orientations of fcc provide similar cross-sections up to 5 atoms.

a) Gaussian Mixture Model

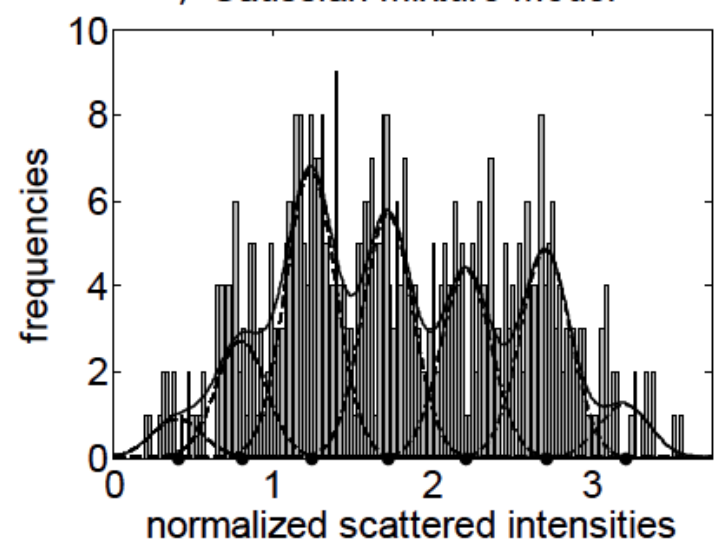

b) Gaussian Mixture Model

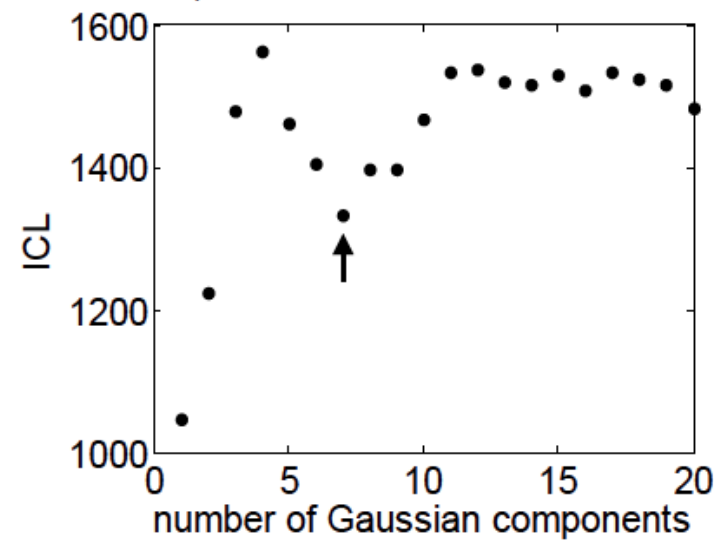

Figure 4 a) Histogram of integrated column intensities from 3 frames (1 frame from each region) with a fitted Gaussian Mixture Model. b) The order criterion selection from the Gaussian Mixture Model. The arrow shows the optimal number of components needed for the Gaussian fitting. The three frames from each region were chosen from the movie (supplementary information) at $\mathrm{t}=680 \mathrm{~s}, 702 \mathrm{~s}, 392 \mathrm{~s}$ for R1, R2 and R3 respectively. 


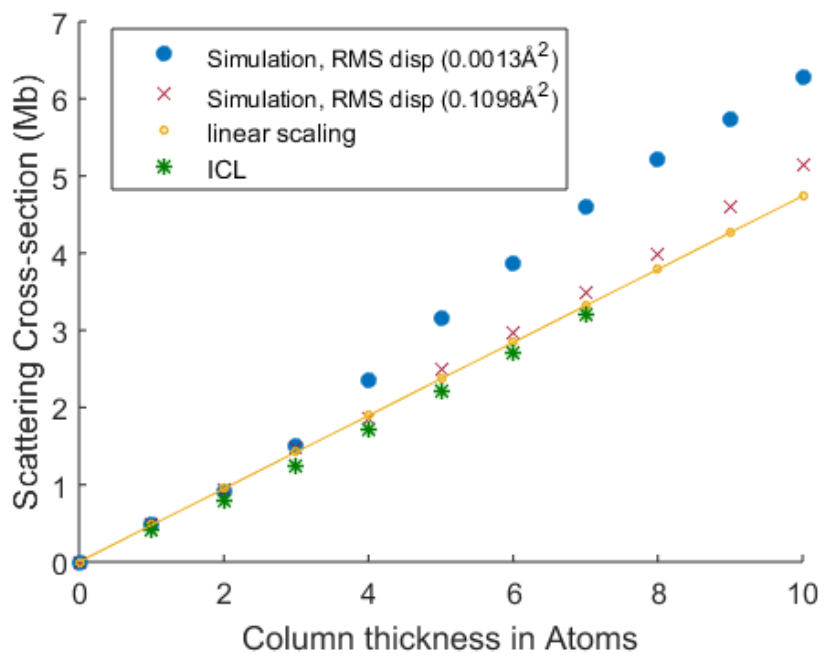

Figure 5 ICL analysis of the integrated intensity as a function of atomic column thickness. The analysis was performed using components from one frame from each of the analysis regions. The figure shows a simulation library (fcc $<110>$ ) corresponding to room temperature (Root Mean Squared (RMS) displacement of $0.0013 \AA^{2}$ ), a fictitious high temperature (Root Mean Squared (RMS) displacement of $0.1098 \AA^{2}$ ), and a linear model where the number of atoms multiplied by the cross-section for a single atom is used. 

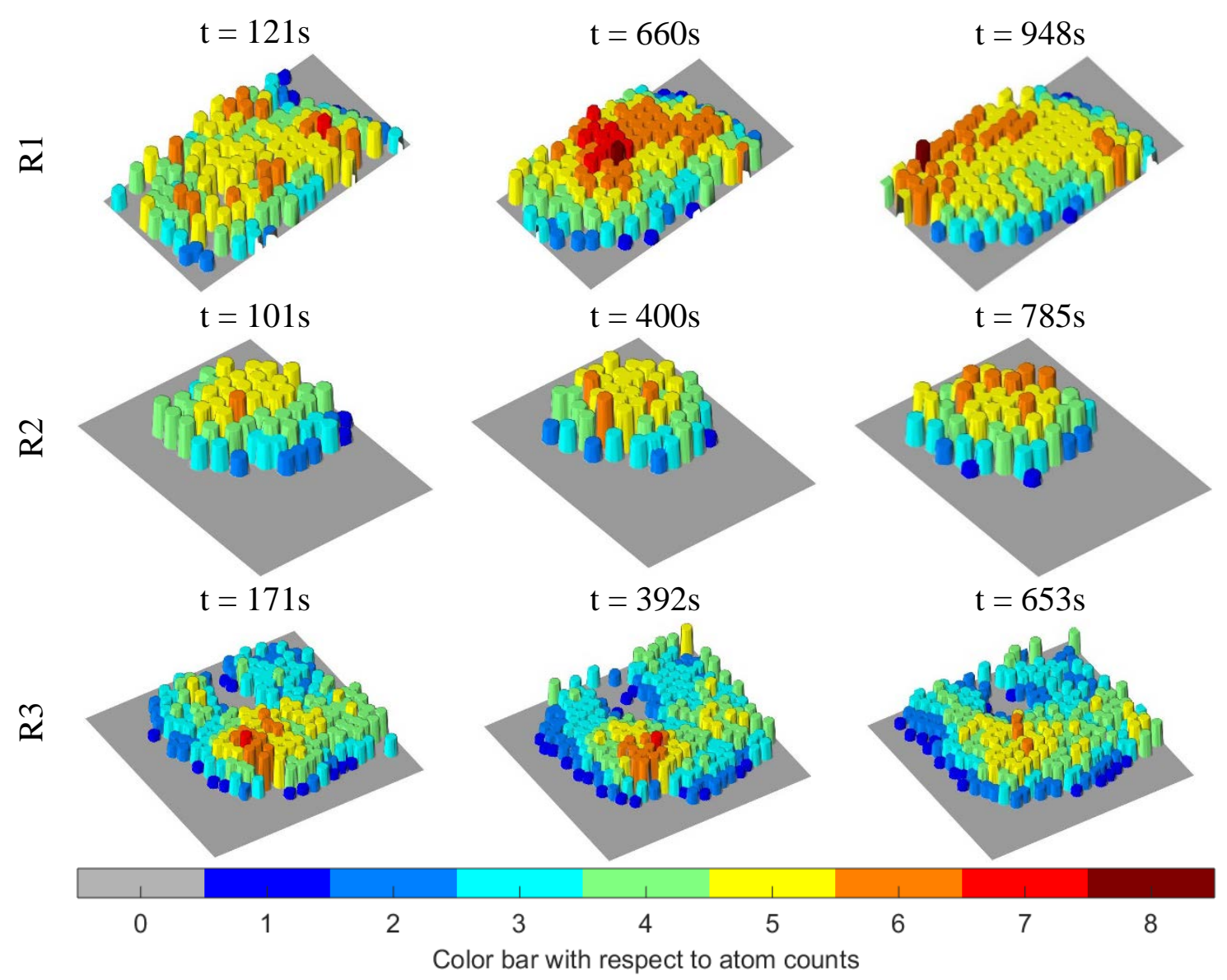

Figure 6. Surface plots created using the linear library from Figure 5. These plots and the average thickness measurements from Figure 7 indicate that structures of stable thicknesses were formed after a few minutes of imaging. 


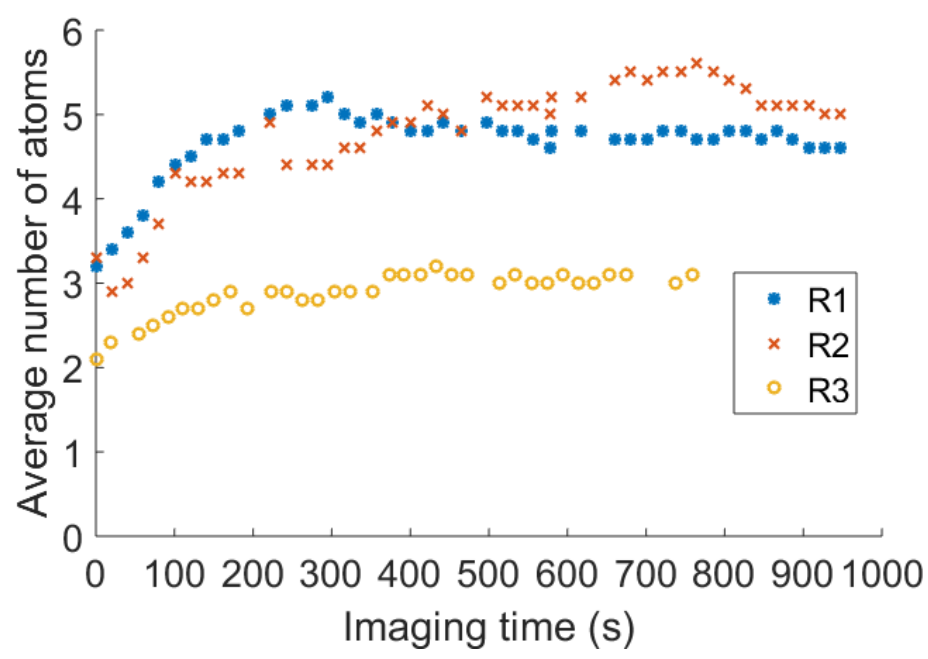

Figure 7. Mean thickness plot as the catalyst was being imaged. All regions stabilised in thickness after being exposed to the electron beam for a while. Time $t=0$ s represents the start of the acquisition process but the materials had been subjected to some electron irradiation ahead of this time. 